\title{
Pump as turbine: dynamic effects in small hydro
}

\author{
Pedro A. Morgado ${ }^{1, *}$, Helena M. Ramos ${ }^{2, * *}$ \\ ${ }^{1,2}$ Civil Engineering Department and CEHIDRO, Instituto Superior Técnico, Technical University of \\ Lisbon, Portugal. \\ *Av. Rovisco Pais, 1049-001, Lisbon, Portugal,E-mail:plmorgado@gmail.com \\ **Av.Rovisco Pais, 1049-001, Lisbon,Portugal,E-mail: helena.ramos@civil.ist.utl.pt
}

\begin{abstract}
This work focuses on the hydraulic aspects of a Pump as Turbine (PAT) when subjected to different working conditions, including accidental situations. This will provide useful information for the choice of the most adequate solution and will ensure a co rrect design and safe operation of the installed equipments. This work is supported by experimental data obtained in a series of tests performed at the university's laboratory and by calibrated computational modelling. The obtained data was used to perform a sensitivity analysis on the relation between discharge variation, runaway time and rotational speed and make inferences about the expected peak overpressure in an accidental situation. Based on the results, the hydropower system may be characterized by a relevant set of calculated parameters that are of extreme utility in the construction and calibration of a computational model capable of accurately predicting different scenarios and alternatives.
\end{abstract}

Keywords: Dynamic effects; Reversible hydro; Safety solutions; Hydraulic transients.

\section{Introduction}

In the operation of hydropower systems it is inevitable the occurrence of variations in the flow, being true either in routine manoeuvres, either in accidental or exceptional unforeseen events. To ensure the safety and reliability throughout the system life, it is very important that these dynamic effects and the associated risk factor are considered in the early stages of each design and the overpressures are accurately estimated $[1,2]$. A detailed analysis for each operating situation is vital for understanding the dynamic behavior of hydro-mechanical equipment and their interaction with the flow and hydraulic circuit. This study is based on a parametric characterization of the different components of a hydro system as a support for a CFD modeling.

\section{Methodology}

\subsection{Simulation-based modelling}

\subsubsection{Method of Characteristics}

The quantitative analysis of unsteady flow through long conveyance systems is based on the fundamental hydrodynamic principles described by the dynamic (Eq. (1)) and continuity (Eq. (2)) equations [3]:

$$
\begin{aligned}
& \frac{\partial V}{\partial t}+g \frac{\partial H}{\partial x}+\frac{f V|V|}{2 D}=0 \\
& \frac{\partial H}{\partial t}+V \frac{\partial H}{\partial x}+\frac{c^{2}}{g} \frac{\partial V}{\partial x}=0
\end{aligned}
$$

The previous equations can be used given the following conditions: the fluid in the pipe is homogeneous and mono-phase; the flow is mainly one-dimensional and the velocity profile is considered uniform through the cross-section of the pipe; the pipe axis remains static; the fluid and the pipe walls have physical linear elastic properties. For each calculation time step, 
each pipe will be divided in a finite number of stretches bounded by the calculation sections building the rectangular mesh presented in Figure 1.

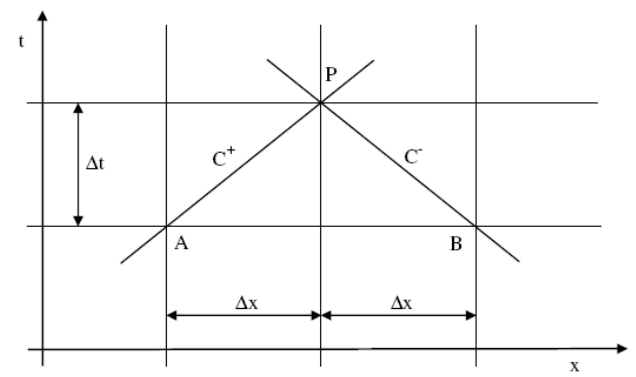

Figure 1 - Method of characteristics calculation grid.

The positive and negative characteristic equations can be written as follows:

$Q_{P}=C_{P}-C_{a} H_{P}$

$Q_{P}=C_{n}+C_{a} H_{P}$

in which:

$$
\begin{aligned}
& C_{P}=Q_{A} \frac{g A}{c} H_{A}-\frac{f \Delta t}{c} Q_{A}\left|Q_{A}\right| \\
& C_{n}=Q_{B} \frac{g A}{c} H_{B}-\frac{f \Delta t}{c} Q_{B}\left|Q_{B}\right|
\end{aligned}
$$

and:

$$
C_{a}=\frac{g A}{c}
$$

Eq. (3) and Eq. (4) are only valid along the positive and negative characteristic lines, respectively. The values of $C_{P}$ and $C_{n}$ are known for each time step, although for the interior points of the mesh there are two unknowns, $Q_{P}$ and $H_{P}$. These values can be determined by solving simultaneously Eq. (3) and Eq. (4):

$Q_{P}=0.5\left(C_{P}+C_{n}\right)$

At both ends of each pipe it is only possible to define one characteristic line, so in order solve the system another equation is needed. This additional information may be obtained through the introduction of a boundary condition. Depending on the simulated system this boundary may be another pipe (with different characteristics), a reservoir, a turbine, a pump, a valve, a protection device or any other component analytically describable.

\subsubsection{Turbine}

The functioning of a turbogenerator can be characterized by a secific valve-type with adapted characteristics associated to customized closure maneuvers. Even though this solution may roughly simulate the vast majority of systems and situations, it does not consider the 
specific parameters of the turbine, nor does it consider the overspeed of the turbine wheel and its complex interaction with the conveyance system. On this matter an innovative formulation was proposed for the simulation of the dynamic behavior of a turbogenerator based on a set of significant parameters that characterize a turbogenerator, allowing the evaluation of overspeed induced extreme upsurges and its propagation along the hydraulic circuit $[4,5,6,7,8,9]$. This methodology simulates the turbine as a hydraulic resistive element, where the head lost by the flow is characterized by the basic formula of a hydraulic orifice equipped with dynamic discharge and rotational speed coefficients:

$Q_{P}=C_{g} Q_{R}\left[1+\frac{\alpha_{R}-1}{\beta_{R}-1}\left(\frac{N}{N_{R}} \sqrt{\frac{H_{R}}{H_{u}}}-1\right)\right] \sqrt{\frac{H_{u}}{H_{R}}}$

in which $q_{P}$ and $h_{P}$ are respectively the relative flow through the orifice and available head at a certain time step. The factor $C_{g}$ is the gate opening coefficient that defines the maximum discharge for a given head and rotational speed, as a function of the gate opening. The factor $C_{S}$ accounts for the runner's rotational speed, adjusting the flow in each time step accordingly with the following formulation:

$$
C_{S}=1+\frac{\alpha_{R}-1}{\beta_{R}-1}\left(\frac{n}{\sqrt{h}}-1\right)
$$

where $n$ stands for the dimensionless value of the rotational speed. The parameters $\alpha_{R}=\frac{Q_{E}}{Q_{0}}$ and $\beta_{R}=\frac{N_{E}}{N_{0}}$ are established for each turbomachine and represent the relation between the runaway situation and rated conditions, respectively for the flow and rotational speed. These values may be obtained from the manufacturers of the turbo equipments. The variation of the rotational speed for each time step depends on the inertia of the rotating masses, $I$, and the equilibrium between the hidraulic torque, $T_{H}$, and the magnetic torque, $T_{M}$. This relation may be expressed through the rotating mass equation:

$\frac{d \omega}{d t}=\frac{60}{2 \pi}\left(T_{H}-T_{M}\right)$

in which $\omega$ is the angular speed (rpm). From Eq. (11), it is possible to conclude that for $T_{M}=$ 0 , the acceleration of the runner depends only on the hydraulic torque $\left(T_{H}\right)$ and the inertia of the rotating masses, $I$. The hydraulic torque actuating in the rated operating conditions is given by the following equation:

$B_{H, 0}=\left(\frac{60}{2 \pi}\right) \frac{\gamma \eta_{0} Q_{0} H_{0}}{N_{0}}$

in wich $\eta_{0}=$ rated efficiency; $\mathrm{Q}_{0}=$ rated flow; $\mathrm{H}_{0}=$ rated net head; $\mathrm{N}_{0}=$ rated runner speed. Assuming a linear variation of the discharge with the rotating speed under runaway conditions, the hydraulic torque at each timestep may obtained by multiplying the initial torque by a corrective factor, $b$, given by the following equation where $e=\frac{\eta}{\eta_{0}}$ : 
$b=\frac{B_{H}}{B_{H, 0}}=h^{3 / 2} C_{g} \frac{e}{n}\left[1-\frac{\frac{n}{\sqrt{h}}-1}{\beta_{R}-1}\right]$

The evaluation of the efficiency is a complex matter that depends on a vast set of parameters. This value may be considered to vary accordingly to the following approximate equations [4, 9].

$$
\begin{cases}\eta_{0} \frac{N}{N_{0}} & \text { for } \mathrm{N}<\mathrm{N}_{0} \\ C_{g}\left(\frac{N_{E}}{N_{E}-N_{0}}-\frac{N}{N_{E}-N_{0}}\right) \eta_{0} & \text { for } \mathrm{N}>\mathrm{N}_{0}\end{cases}
$$

\subsubsection{Pumping system}

The pump operation may be accurately simulated using the method of characteristics incorporating specific boundary conditions. Pump manufacturers generally provide the characteristic curves for their pumps as a function of the wheel diameter. These curves relate flow, manometric head, efficiency and power for a s pecific rotational speed and can be approximately represented in the first quadrant of operation $(\mathrm{H}, \mathrm{Q})$ by a second degree polynomial (Eq. (15)) [10]. Should this methodology be applied, a check valve should be installed at the downstream pump section to avoid reverse flow.

$H_{0}=A N^{2}+B N Q-C Q^{2}$

The values $\mathrm{A}, \mathrm{B}$ and $\mathrm{C}$ are constants and can be estimated from three known pairs of values $(Q, H)$ of the pump characteristic curve. If correct data is available, a h igher degree polynomial may be considered to enable a multi operating zone simulation. Although pump characteristic curves in the pumping zone operation are usually easy to obtain, the same is not true for the remaining zones. Figure 2 presents a schematic of a pump with the upstream and downstream connecting pipes and hydraulic grade line.

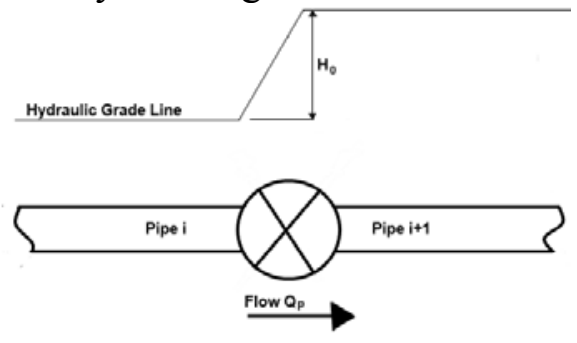

Figure 2 - Schematic drawing of a pump.

Referring to Figure 2 and considering the continuity equation, the relation, $Q_{P, i}=Q_{P, i+1}$, can be written. If the losses at the junctions are neglected, Eq. (16) gives the relation between head and discharge, in which $N$ is the rotational speed:

$H_{P, i+1}-H_{P, i}=A N^{2}+B N Q-C Q^{2}$

when $A_{i}=A_{i+1}$, substituting Eqs. (3) and (4) into Eq. (16) the following polynomial is obtained, which can be solved for $Q_{P}$. 


$$
C C_{a} Q_{P}^{2}+\left(B N C_{a}-2\right) Q_{P}+\left(A C_{a} N^{2}+C_{n}+C_{P}\right)=0
$$

Once the flow $Q_{P}$ is known, the heads $H_{P, i}$ and $H_{P, i+1}$ can be calculated for each time step. The total or partial shutdown of the pump as the cause of severe transient regimes may also be simulated. In this case the variation of the runner speed is given by Eq. (11) in which the hydraulic torque can be calculated based on efficiency data provided by the manufacturer.

\subsubsection{Valve}

Valves are installed in hydro systems to regulate the flow (or the pressure) by opening, closing or partially obstructing the pipe section. They can also be utilized to isolate system components in order to enable maintenance procedures or even their replacement. Figure 3 presents the schematic of a valve in hydraulic ciruit.

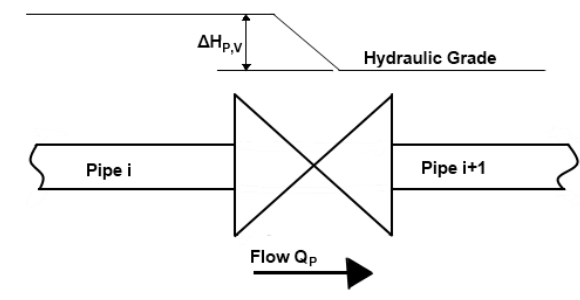

Figure 3 - Schematic of a generic valve.

The boundary condition for a valve can be written based on steady state head loss equation. The flow through the valve, $Q_{P}$, remains constant and the head loss can be written as the difference between the head at upstream and downstream valve section:

$$
\Delta H_{P, V}=H_{P, i}-H_{P, i+1}=K_{V} \frac{Q_{P}^{2}}{2 A g}
$$

in which $\mathrm{K}_{\mathrm{V}}$ is a head loss coefficient obtained experimentally that depends on the Reynolds number, the type of valve and its opening at each time step. Substituting Eq. (3) or (4) in Eq. (18) a second degree polynomial equation is obtained:

$$
\frac{K_{V} C_{a}}{2 g A^{2}} Q_{P}^{2}+2 Q_{P}-\left(C_{P}+C_{n}\right)=0
$$

Solving for $\mathrm{Q}_{\mathrm{P}}$, the flow through the valve is obtained for each instant of simulation and subsequently the upstream and downstream head. For the situation where the valve is fully open, the head loss may be neglected and the flow can be determined by solving Eq. (8) or a fixed value should be considered. For the determination of the parameter $K_{V}$, a s et of empirical results for each type of valve is presented in [11].

\subsection{Model validation}

Some supporting experimental tests were carried out in the Hydraulic lab of DECivil/IST, in order to analyze the behaviour of a reaction type turbomachine in different operation conditions. The hydraulic circuit is composed by a pipe of High-density polyethylene (HDPE), with an internal diameter of $0.043 \mathrm{~m}$ and a length of $100 \mathrm{~m}$, which connects the pressurized vessel to the turbomachine. At the downstream end the water is discharged into a free surface tank. Both the air vessel and the free surface reservoir are responsible for 
imposing a constant head respectively on the upstream and downstream section. An intercalated pump is responsible for setting a recirculated constant flow. The turbomachine is a horizontal, single-stage pump-as-turbine, PAT, KSB model Etanorm 32-125. The generator was connected to the national grid via a 1 arge electrical transformer. Figure 4 presents a comparison between experimental pressure head variation and the corresponding simulation in the event of a full load rejection followed by a valve actuation.
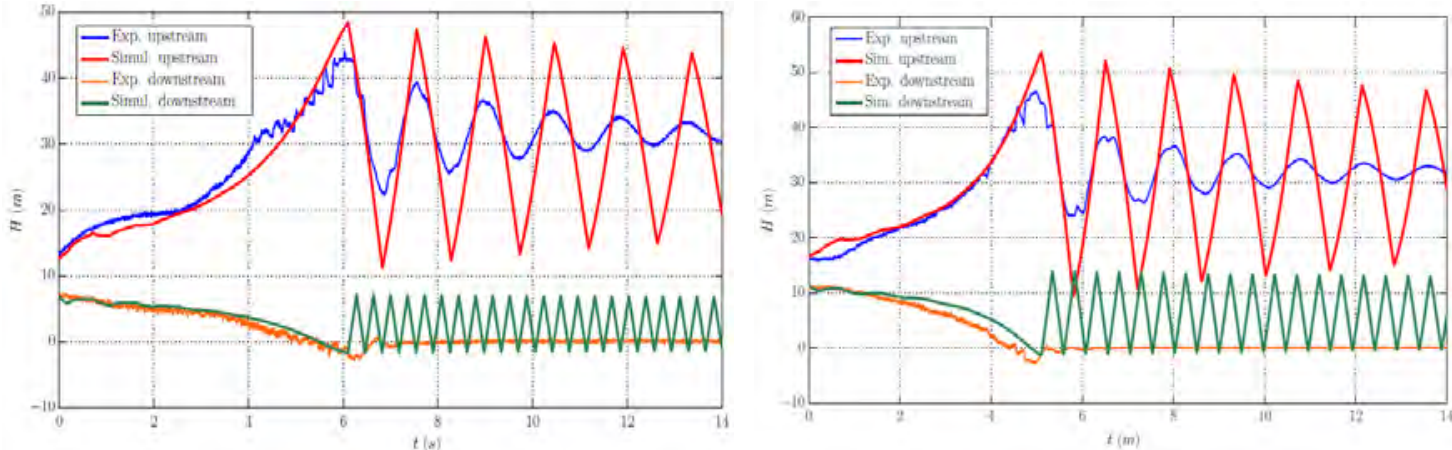

Figure 4 - Comparison between simulation and experimental results for $Q=4.2 \mathrm{l} / \mathrm{s}$ and $Q=3.9 \mathrm{l} / \mathrm{s}$

\section{Overspeed and dynamic effects}

Based on a general hydropower system, a detailed analysis of the runaway effect without the interference of the closure of the flow control shutter (e.g. guidevane) or any special protection device was established. This was done in order to fully isolate and better understand the phenomenon under analysis. Under these conditions several independent computer simulations were carried out for a broad set of pairs of values of inertia, I, and specific speed, $\mathrm{n}_{\mathrm{S}}$. Figure 5 shows the upsurge on the upstream section of a turbine for the different combinations of parameters. For the lower values of $n_{S,}$ the overspeed results in a flow reduction, causing an upsurge in the hydraulic system. On the other hand, turbines with high specific speeds are associated to an increase in flow when a load rejection occurs, resulting in sub-atmospheric pressures in the pipeline.

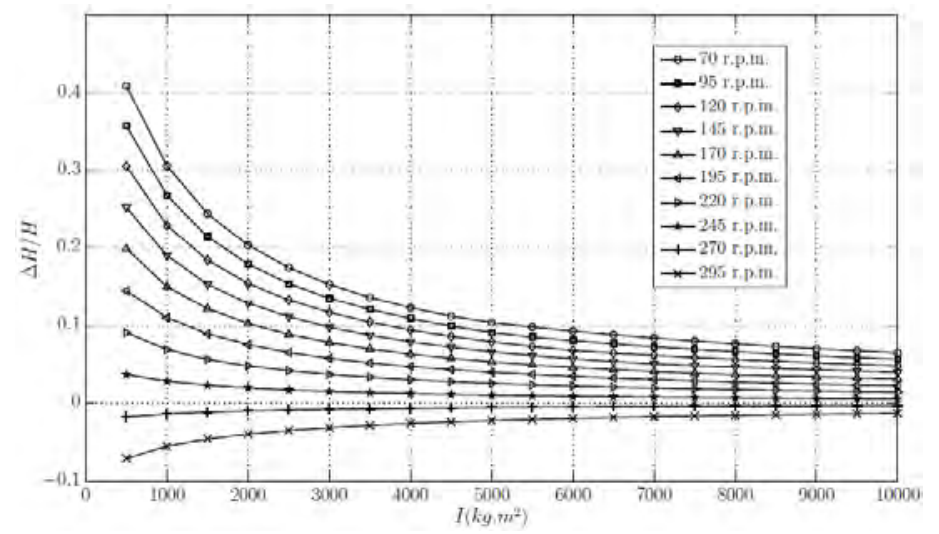

Figure 5 - Extreme relative pressure head as a function of I and $n_{S}$.

\section{Load rejection and closure of flow control device}

From the different emergency operations a hydropower system endures throughout its service life, the full load rejection followed by the actuation of a discharge shutter may stand among the most severe situations. Considering the same turbomachine used in the paragraph 3 , the influence of the length, L, of the pipeline and its interaction with the safety valve closure time, $t_{C}$, was studied. For this purpose, a set of simulations were carried out in order to 
calculate the maximum upsurge for different combinations of values, obtaining the results presented in Figure 6.

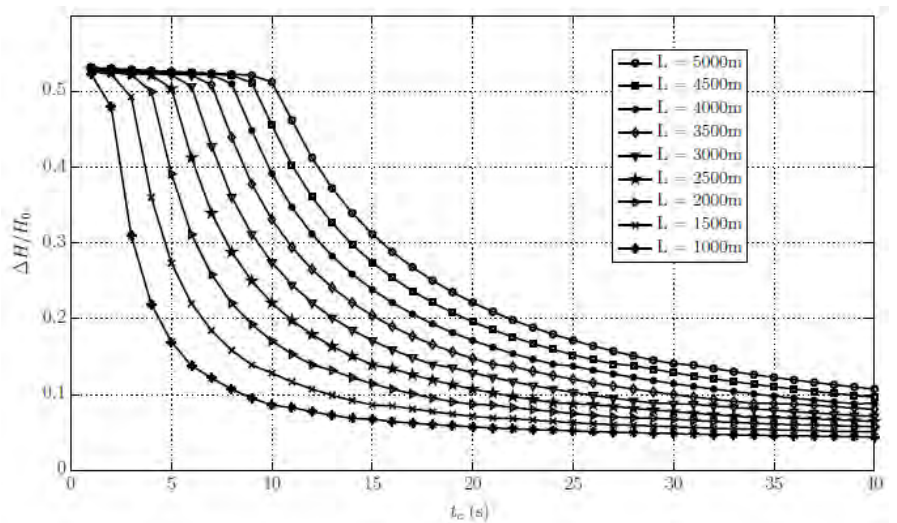

Figure 6 - Maximum relative overpressure for different combinations of $L$ and $t_{C .}$

For all pipeline lengths, when approaching the elastic time constant, $T_{E}$, the maximum values of $\Delta H$ rise very quickly. It should be noted that below a certain value of $t_{C}$, for each different penstock length, all the curves tend to a common maximum value. This is the domain of what is called a fast manoeuvre, $t_{C}<T_{E}$, in which the transient peak overpressure depends only on the celerity of the elastic waves, c, and the mean flow velocity, $U_{0}$, given by the FriselJoukowsky formula.

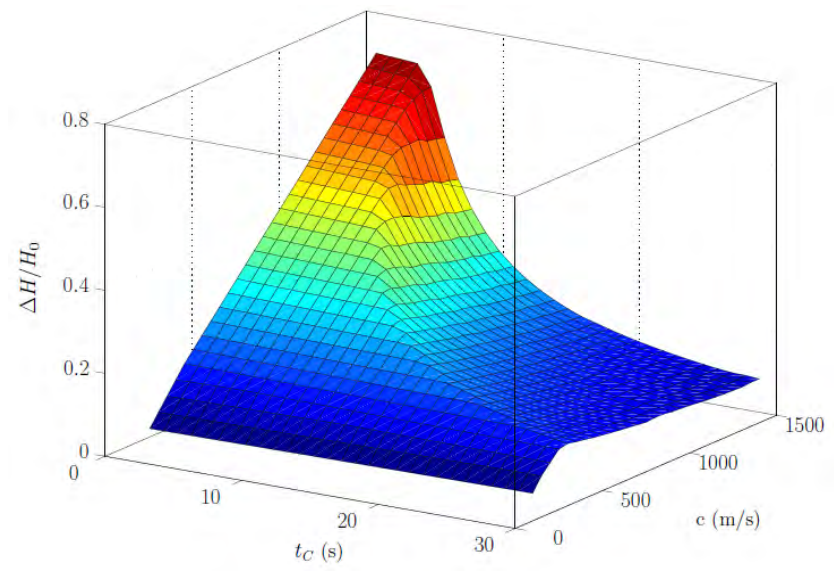

Figure 7 - Maximum relative overpressure for different combinations of $c$ and $t_{C}$

Figure 7 was generated by utilizing the developed computational model, to show the maximum overpressure reached for different combinations of values of the celerity, $\mathrm{c}$, and closure durations, $t_{C}$, for a fixed penstock length $L=4000 \mathrm{~m}$. When observing closely, it becomes evident that the classification of a closure manoeuvre as fast $\left(\mathrm{t}_{\mathrm{C}}<\mathrm{T}_{\mathrm{E}}\right)$ or slow $\left(\mathrm{t}_{\mathrm{C}}>\right.$ $\mathrm{T}_{\mathrm{E}}$ ) is a relative concept that depends on the celerity of the elastic waves, thus on the pipe properties. In fact, the lower the value of $c$, the bigger the value of $t_{C}$ must be in order to perform a slow closure manoeuvre. For a slow manoeuvre, the maximum pressure head depends essentially on the mean fluid velocity and on the flow control valve closure time. The maximum value can be estimated based on the by Michaud formula. Unlike what happens in a fast manoeuvre, the maximum overpressure is independent from the wave speed celerity.

\section{Results}

Both the experimental and the simulation results demonstrate the turbine load variation, in particular the runaway condition has a relevant impact on the discharge and, given the right circumstances, can be the sole cause of severe transients in hydropower systems. This is 
especially evident for certain combinations of values of $n_{S}$ and $I$ that can result in a significant flow reduction during a short time interval. The comparison between the developed model and the experimental test results suggests a good estimation for the maximum transient overpressures, presenting conservative values valid for hydro and pumping design. This formulation based on modular linkable components proved to be flexible enough to allow the easy setup of a $\mathrm{v}$ ast set of different scenarios that are able to simulate the majority of situations. This methodology has the ability of predicting the runner overspeed of turbogenerators, as well as pumping stoppage and its effect on the flow along the pipe system.

\section{References}

[1] Ramos, H., Guidelines for Design of Small Hydropower Plants. Book published by WREAN (Western Regional Energy Agency and Network) and DED (Department of Economic Development - Energy Division). Número de páginas: 205. Belfast, North Ireland. ISBN 972-96346-4-5, 2000.

[2] Morgado, P.; Ramos, H., "Renewable Energy Production Integrated in Water Supply Systems: Dynamic Effects Analysis" in Seminario Iberoamericano sobre Planificación, Proyecto y Operación de Sistemas de Abastecimiento de Agua, Valência (Espanha), 2427 de Novembro de 2009 (in Portuguese).

[3] Streeter, VL e Wylie, EB, Hydraulic Transients. New York, McGraw-Hill Book Co., 1967.

[4] Ramos, H., Simulation and Control of Hydraulic Transients in Small Hydro. Modelling and Analysis of Induced Effects by Turbogenerator Overspeed (in Portuguese). Ph. D. Dissertation in Civil Engineering. Portugal, Instituto Superior Técnico, 1995.

[5] Ramos, H.; Almeida A.B, Experimental and Computational Analysis of Hydraulic Transients Induced by Small Reaction Turbomachines (in Portuguese). APRH, LNEC. Lisboa, 2001.

[6] Ramos, H., Unconventional Dynamic Effects in Pressurized Hydraulic System (in Portuguese). Support document for the subject Transients in Elevation and Hydroelectric Systems from the PhD in Hydraulics and Water Resources. IST, DECivil, 2004.

[7] Ramos, H. and Almeida, A. B., Parametric Analysis of Waterhammer Effects in Small Hydropower Schemes. HY/1999/021354. ASCE - Journal of Hydraulic Engineering. Volume 128, 7, pp. 689-697, ISSN 0733-9429, 2002.

[8] Ramos, H; Almeida, A. B., Dynamic orifice model on waterhammer analysis of high and medium heads of small hydropower schemes. Journal of Hydraulic Research, IAHR, Vol. 39 (4), pp. 429-436, ISSN-0022-1686, 2001.

[9] Morgado, P.; Ramos, H., "Dynamic Effects Analysis in Hydropower Systems" (in Portuguese), $10^{\circ}$ Congresso da Água (Portugal), 2010

[10] Ramos, H., Support document for the subject Elevation and Hydroelectric Systems Subject of the Hydraulic and Water Resource Msc. IST, DECivil, 2003.

[11] Lencastre, A. - General Hydraulic (in Portuguese). Hidroprojecto, 1983. 